Proyecciones Journal of Mathematics Vol. 36, N ${ }^{o}$ 1, pp. 13-27, March 2017.

Universidad Católica del Norte

Antofagasta - Chile

\title{
Yet another variant of the Drygas functional equation on groups
}

\author{
Prasanna K. Sahoo \\ University of Louisville, U.S.A. \\ Received: August 2016. Accepted : August 2016
}

\begin{abstract}
Let $G$ be a group and $\mathbf{C}$ the field of complex numbers. Suppose $\sigma_{1}, \sigma_{2}: G \rightarrow G$ are endomorphisms satisfying the condition $\sigma_{i}\left(\sigma_{i}(x)\right)=x$ for all $x$ in $G$ and for $i=1,2$. In this paper, we find the central solution $f: G \rightarrow \mathbf{C}$ of the equation $f(x y)+f\left(\sigma_{1}(y) x\right)=$ $2 f(x)+f(y)+f\left(\sigma_{2}(y)\right)$ for all $x, y \in G$ which is a variant of the Drygas functional equation with two involutions. Further, we present a generalization the above functional equation and determine its central solutions. As an application, using the solutions of the generalized equation, we determine the solutions $f, g, h, k: G \times G \rightarrow \mathbf{C}$ of the functional equation $f(p r, q s)+g(s p, r q)=2 f(p, q)+h(r, s)+k(s, r)$ when $f$ satisfies the condition $f(p r, q s)=f(r p, s q)$ for all $p, q, r, s \in G$.
\end{abstract}

Keywords and phrases : Drygas functional equation; group; Fréchet's functional equation; involution; semigroup, Whitehead functional equation.

Subjclass : 2010 Mathematics Subject Classification. Primary 39B52. 


\section{Introduction}

The functional equation

$$
f(x y)+f\left(x y^{-1}\right)=2 f(x)+2 f(y)
$$

for all $x, y \in G$, where $G$ is a group written multiplicatively and $y^{-1}$ is the inverse of $y$, is known as the quadratic functional equation and it serves in certain abstract spaces for the definition of norm. It was studied by many authors including Jensen [8, 9], Jordan and von Neumann [10], Kurepa [14], Aczél and Vincze [2], Aczél [1], Kannappan [11, 12, 13], and Yang [24]. Sinopoulos [21] considered the following generalization of quadratic functional equation

$$
f(x y)+f(x \sigma(y))=2 f(x)+2 f(y)
$$

for all $x, y \in S$, where $S$ is a commutative semigroup and $\sigma: S \rightarrow S$ is an endomorphism of $S$ such that $\sigma(\sigma(x))=x$ for all $x \in S$. In [4], a variant of the quadratic functional equation, namely

$$
f(x y)+f(\sigma(y) x)=2 f(x)+2 f(y)
$$

for all $x, y \in G$, where $G$ is a group (not necessarily abelian) was considered. If $G$ is an abelian group or $f$ is a central function on group $G$, then the equations (1.1) and (1.2) are equivalent. The following functional equation is a variant of Drygas functional equation:

$$
f(x y)+f(\sigma(y) x)=2 f(x)+f(y)+f(\sigma(y))
$$

for all $x, y \in G$. If $f$ satisfies $f(\sigma(y))=f(y)$ for all $y \in G$, then (1.2) and (1.3) are equivalent. This equation was studied by the author on groups in [18]. The survey paper [7] and the references therein contain a wealth of information on quadratic functional equation and Drygas functional equation. The inspiration for studying the functional equation (1.4) came from the paper [23]. The functional equation

$$
f(x y)+f\left(\sigma_{1}(y) x\right)=2 f(x)+f(y)+f\left(\sigma_{2}(y)\right), \quad \forall x, y \in G
$$

with two involutive endomorphisms is a generalization of (1.3). The functional equation (1.4) is a more general equation on an abelian group $G$ than (1.3) since choosing $\sigma_{1}$ and $\sigma_{2}$ appropriately in (1.4) one can obtain (1.2) and (1.3). 
This paper is organized as follows: In Section 2, we introduce the definition of relevant terminologies and notations that will be used in the subsequent sections of the paper. In Section 3, we prove some preliminary results that will be used to determine the solution of the equation (1.4) on groups. Section 4 contains the solution of (1.4) on groups when $f$ is a central function. In Section 5, we generalize the functional equation (1.4) and determine its central solutions. As an application, using the solutions of a generalization of (1.4), we determine the central solutions of a functional equation (6.3) related to stochastic distance measures.

\section{Notation and terminology}

Let $G$ be a group and $S$ be a monoid written multiplicatively. Thus $S$ is a semigroup with the identity element $e$. Let $\mathbf{C}$ be field of complex numbers. A function $f: S \rightarrow \mathbf{C}$ is said to be central if and only if $f(x y)=f(y x)$ for all $x, y$ in $S$ (see [22]). In particular, a function $f: S \times S \rightarrow \mathbf{C}$ is said to be central if and only if $f(x y, u v)=f(y x, v u)$ for all $x, y, u, v \in S$. This says that $f$ is central on the product monoid $S \times S$. A function $A: S \rightarrow \mathbf{C}$ is said to be a homomorphism (or an additive function) if $A(x y)=A(x)+A(y)$ for all $x, y \in S$. It is known that $A(e)=0$. In particular, $A: S \times S \rightarrow \mathbf{C}$ is said to be a homomorphism if $A(x y, u v)=$ $A(x, u)+A(y, v)$ for all $x, y, u, v \in S$. It is known that $A(e, e)=0$. A function $B: S \times S \rightarrow \mathbf{C}$ is said to be a bihomomorphism if and only if $B$ is a homomorphism in each variable, that is $B(x y, z)=B(x, z)+B(y, z)$ and $B(x, y z)=B(x, y)+B(x, z)$ for all $x, y, z \in S$ or equivalently $B$ satisfies $B(x y, u v)=B(x, u)+B(x, v)+B(y, u)+B(y, v)$ for $x, y, u, v \in S$. The map $B: S \times S \rightarrow \mathbf{C}$ is said to be symmetric if and only if $B(x, u)=B(u, x)$ for all $x, u \in S$. It can be easily seen that $B(e, x)=B(x, e)=B(e, e)=0$ for all $x \in S$ and $B\left(x^{-1}, u\right)=-B(x, u)$ for $x, u \in G$. The map $\sigma: S \rightarrow S$ is an endomorphism satisfying $\sigma(\sigma(x))=x$ for all $x \in S$. Such a function $\sigma$ will be called an involutive endomorphism of $S$. The set of all homomorphisms from $G$ to the additive group of the complex numbers $\mathbf{C}$ will be denoted by $\operatorname{Hom}(G, \mathbf{C})$. Similarly, the set of all symmetric bihomomorphisms from $G \times G$ to the additive group of the complex numbers $\mathbf{C}$ will be denoted by $\operatorname{SBihom}(G \times G, \mathbf{C})$.

A function $f: S \rightarrow \mathbf{C}$ called $\sigma$-even if and only if $f(\sigma(x))=f(x)$ for all $x \in S$. Similarly, a function $f: S \rightarrow \mathbf{C}$ called $\sigma$-odd if and only if $f(\sigma(x))=-f(x)$ for all $x \in S$. A function $f: S \rightarrow \mathbf{C}$ is $\sigma_{2} \circ \sigma_{1}$-even if and only if $f\left(\sigma_{1}(x)\right)=f\left(\sigma_{2}(x)\right)$ for all $x \in S$. 


\section{Some preliminary results}

Let $D(S, \mathbf{C})$ denote the set of all solutions $f: S \rightarrow \mathbf{C}$ of the functional equation

$$
f(x y)+f\left(\sigma_{1}(y) x\right)=2 f(x)+f(y)+f\left(\sigma_{2}(y)\right)
$$

for all $x, y \in S$ and $W(S, \mathbf{C})$ denote the set of all solutions $f: S \rightarrow \mathbf{C}$ of the functional equation

$$
f(x y z)=f(x y)-f(z)+f(y z)-f(x)+f(x z)-f(y)
$$

for all $x, y, z \in S$.

Lemma 3.1. Let $S$ be a monoid and $\sigma_{1}, \sigma_{2}: S \rightarrow S$ be endomorphisms satisfying $\sigma_{i}\left(\sigma_{i}(x)\right)=x$ for all $x \in S$ and for $i=1,2$. If $f \in D(S, \mathbf{C})$, then $f \in W(S, \mathbf{C})$.

Proof. Let $f \in D(S, \mathbf{C})$, that is, $f$ be a solution of (1.4). Then, it is easy to check that $f(e)=0$. Replacing $x$ by $x y$ and $y$ by $z$ in (1.4) we get that

$$
f(x y z)+f\left(\sigma_{1}(z) x y\right)=2 f(x y)+f(z)+f\left(\sigma_{2}(z)\right)
$$

for all $x, y, z \in S$. Similarly, replacing $x$ by $\sigma_{1}(z) x$ in (1.4) and using the fact that $\sigma_{2}$ is an endomorphism, the resulting equation yields

$$
f\left(\sigma_{1}(z) x y\right)+f\left(\sigma_{1}(y z) x\right)=2 f\left(\sigma_{1}(z) x\right)+f(y)+f\left(\sigma_{2}(y)\right)
$$

for all $x, y, z \in S$. Replacing $y$ by $y z$ in (1.4) and rewriting we get that

$$
f\left(\sigma_{1}(y z) x\right)=2 f(x)+f(y z)+f\left(\sigma_{2}(y z)\right)-f(x y z)
$$

for all $x, y, z \in S$. Next, replacing $y$ by $z$ in (1.4), we have

$$
f\left(\sigma_{1}(z) x\right)=2 f(x)+f(z)+f\left(\sigma_{2}(z)\right)-f(x z)
$$

for all $x, y, z \in S$. Subtracting (3.3) from (3.2), we obtain

$$
\begin{aligned}
& f(x y z)-f\left(\sigma_{1}(y z) x\right) \\
& =2 f(x y)+f(z)+f\left(\sigma_{2}(z)\right)-2 f\left(\sigma_{1}(z) x\right)-f(y)-f\left(\sigma_{2}(y)\right) .
\end{aligned}
$$

Using (3.4) and (3.5) in (3.6) and simplifying the resulting expression, we see that 


$$
\begin{aligned}
& f\left(\sigma_{2}(y z)\right)+f(y z)-2 f(x y z)+2 f(x y)+2 f(x z) \\
& =2 f(x)+f(y)+f\left(\sigma_{2}(y)\right)+f(z)+f\left(\sigma_{2}(z)\right)
\end{aligned}
$$

for all $x, y, z \in S$. Letting $x=e$ in the previous equation and simplifying, we have

$$
f(y z)-f\left(\sigma_{2}(y z)\right)=f(y)-f\left(\sigma_{2}(y)\right)+f(z)-f\left(\sigma_{2}(z)\right)
$$

for all $y, z \in S$. Defining $A: S \rightarrow \mathbf{C}$ by

$$
A(x):=f(x)-f\left(\sigma_{2}(x)\right)
$$

for all $x \in S$, we obtain from (3.8) $A(y z)=A(y)+A(z)$ for all $y, z \in S$. That is $A: S \rightarrow \mathbf{C}$ is an additive function. Therefore from (3.9), we see that

$$
f\left(\sigma_{2}(x)\right)=f(x)-A(x)
$$

for all $x \in S$. Inserting (3.10) in (3.7) and simplifying we have the asserted equation:

$$
f(x y z)=f(x y)-f(z)+f(y z)-f(x)+f(x z)-f(y)
$$

for all $x, y, z \in S$. Hence $f \in W(S, \mathbf{C})$ and this completes the proof.

Remark 3.2. The equation (3.1) has been referred to as Whitehead functional equation by Faiziev and Sahoo in [6]. In 1950, Whitehead [25] found the solutions of (3.1) on abelian groups. In 2013, Ng and Zhao [15] gave the solution of the equation (3.1) on free groups. If $f$ is a central function, then Whitehead equation takes the form

$$
f(x y z)=f(x y)-f(z)+f(y z)-f(x)+f(z x)-f(y) .
$$

The above equation is often referred to as Fréchet's functional equation. In [11], Kannappan determined the solution of Fréchet's equation on groups.

The next lemma follows from Kannappan's work (see [11], [12], and [13]).

Lemma 3.3. Let $G$ be a group and $f: G \rightarrow \mathbf{C}$ satisfy Fréchet's functional equation

$$
f(x y z)=f(x y)-f(z)+f(y z)-f(x)+f(z x)-f(y)
$$


for all $x, y, z \in G$. Then $f$ is given by

$$
f(x)=a(x)+b(x, x)
$$

for all $x \in G$, where $a \in \operatorname{Hom}(G, \mathbf{C})$ and $b \in \operatorname{SBihom}(G \times G, \mathbf{C})$.

\section{The central solution of the equation (1.4)}

In this section, we determine the central solutions of the functional equation (1.4).

Theorem 4.1. Let $G$ be a group and $\sigma_{1}, \sigma_{2}: G \rightarrow G$ be endomorphisms satisfying $\sigma_{i}\left(\sigma_{i}(x)\right)=x$ for all $x \in G$ and for $i=1,2$. Let $f: G \rightarrow \mathbf{C}$ be a central function satisfying the functional equation (1.4) for all $x, y \in G$. Then

$$
f(x)=A(x)+B(x, x),
$$

where $A \in \operatorname{Hom}(G, \mathbf{C})$ and $B \in \operatorname{SBihom}(G \times G, \mathbf{C})$ satisfying

$$
B\left(\sigma_{1}(x), y\right)=-B(x, y)
$$

and

$$
B\left(\sigma_{2}(y), \sigma_{2}(y)\right)=B(y, y) \quad \text { and } \quad A\left(\sigma_{1}(y)\right)=A\left(\sigma_{2}(y)\right)
$$

for all $x, y \in G$. The converse is also true.

Proof. It is easy to check that any function of the form (4.1) where $A \in \operatorname{Hom}(G, \mathbf{C})$ and $B \in \operatorname{SBihom}(G \times G, \mathbf{C})$ satisfy (4.2) and (4.3) for all $x, y \in G$ is a solution of the equation (1.4). Next, we show that (4.1) is the only solution of (1.4) satisfying (4.2) and (4.3).

Let $f$ be a solution of (1.4) for all $x, y \in G$. Then letting $x=e$ and $y=e$ in (1.4), we see that $f(e)=0$. Further, letting $x=e$, we have

$$
f(y)=f\left(\sigma_{2}\left(\sigma_{1}(y)\right)\right)
$$

for all $y \in G$. Thus $f$ is $\sigma_{2} \circ \sigma_{1}$-even.

Since $f \in D(G, \mathbf{C})$, then from Lemma 3.1 we have that $f \in W(G, \mathbf{C})$, that is $f$ is a solution of the Whitehead functional equation

$$
f(x y z)=f(x y)-f(z)+f(y z)-f(x)+f(x z)-f(y)
$$


for all $x, y, z \in G$. Using the fact that $f$ is central, the previous equation can be written as the Fréchet's functional equation

$$
f(x y z)=f(x y)-f(z)+f(y z)-f(x)+f(z x)-f(y)
$$

for all $x, y, z \in G$. From Lemma 3.3 we have that

$$
f(x)=A(x)+B(x, x)
$$

where $A: G \rightarrow \mathbf{C}$ is a homomorphism and $B: G \times G \rightarrow \mathbf{C}$ is a symmetric bihomomorphism. Using the form of $f$ in (4.5) in the equation (1.4), we obtain

$$
\begin{aligned}
& A(x y)+A\left(\sigma_{1}(y) x\right)-2 A(x)-A(y)-A\left(\sigma_{2}(y)\right) \\
& +B(x y, x y)+B\left(\sigma_{1}(y) x, \sigma_{1}(y) x\right) \\
& -2 B(x, x)-B(y, y)-B\left(\sigma_{2}(y), \sigma_{2}(y)\right)=0
\end{aligned}
$$

for all $x, y \in G$. Using the properties of the homomorphism $A$ and the symmetric bihomomorphism $B$ in the above equation and then simplifying the resulting expression, we have

$$
\begin{aligned}
& A\left(\sigma_{1}(y)\right)+2 B(x, y)+2 B\left(x, \sigma_{1}(y)\right) \\
& +B\left(\sigma_{1}(y), \sigma_{1}(y)\right)-A\left(\sigma_{2}(y)\right)-B\left(\sigma_{2}(y), \sigma_{2}(y)\right)=0
\end{aligned}
$$

for all $x, y \in G$. Since $f$ is $\sigma_{2} \circ \sigma_{1}$-even, therefore $f\left(\sigma_{1}(y)\right)=f\left(\sigma_{2}(y)\right)$ and using (4.1), we obtain

$$
A\left(\sigma_{1}(y)\right)+B\left(\sigma_{1}(y), \sigma_{1}(y)\right)=A\left(\sigma_{2}(y)\right)+B\left(\sigma_{2}(y), \sigma_{2}(y)\right)
$$

for all $y \in G$. Use of (4.7) in (4.6) yields

$$
B\left(x, \sigma_{1}(y)\right)=-B(x, y)
$$

for all $x, y \in G$. Hence, since $B$ is symmetric, we have

$$
B\left(\sigma_{1}(x), \sigma_{1}(y)\right)=-B\left(\sigma_{1}(x), y\right)=-B\left(y, \sigma_{1}(x)\right)=B(y, x)=B(x, y)
$$

for all $x, y \in G$ and thus

$$
B\left(\sigma_{1}(y), \sigma_{1}(y)\right)=B(y, y)
$$

for all $y \in G$. Replacing $y$ by $y^{n}$ for $n \in \mathrm{N}$ (the set of natural numbers) in (4.7), we get

$$
n\left[A\left(\sigma_{1}(y)\right)-A\left(\sigma_{2}(y)\right)\right]+n^{2}\left[B\left(\sigma_{1}(y), \sigma_{1}(y)\right)-B\left(\sigma_{2}(y), \sigma_{2}(y)\right)\right]=0
$$


for all $n \in \mathbf{N}$ and $y \in G$. Hence, we have $A\left(\sigma_{1}(y)\right)=A\left(\sigma_{2}(y)\right)$ and $B\left(\sigma_{2}(y), \sigma_{2}(y)\right)=B\left(\sigma_{1}(y), \sigma_{1}(y)\right)$ for all $y \in G$. Using (4.9), we obtain

$$
B\left(\sigma_{2}(y), \sigma_{2}(y)\right)=B(y, y) \quad \text { and } \quad A\left(\sigma_{1}(y)\right)=A\left(\sigma_{2}(y)\right)
$$

for all $y \in G$. The proof of the theorem is now complete.

The next corollary follows from the last theorem.

Corollary 4.2. Let $G$ be a group and $\sigma: G \rightarrow G$ be an involutive endomorphism. Let the central function $f: G \rightarrow \mathbf{C}$ satisfy the functional equation

$$
f(x y)+f(\sigma(y) x)=2 f(x)+f(y)+f(\sigma(y))
$$

for all $x, y \in G$. Then

$$
f(x)=A(x)+B(x, x),
$$

where $B \in \operatorname{SBihom}(G \times G, \mathbf{C})$ satisfying $B(\sigma(x), y)=-B(x, y)$ for all $x, y \in G$ and $A \in \operatorname{Hom}(G, \mathbf{C})$. The converse is also true.

The next two corollaries also follow from the last theorem.

Corollary 4.3. Let $G$ be a group and $\sigma: G \rightarrow G$ be an involutive endomorphism. Let the central function $f: G \rightarrow \mathbf{C}$ satisfy the functional equation

$$
f(x y)+f(\sigma(y) x)=2 f(x)+2 f(y)
$$

for all $x, y \in G$. Then

$$
f(x)=A(x)+B(x, x),
$$

where $B \in \operatorname{SBihom}(G \times G, \mathbf{C})$ satisfying $B(\sigma(x), y)=-B(x, y)$ for all $x, y \in G$ and $A \in \operatorname{Hom}(G, \mathbf{C})$ satisfying $A(\sigma(x))=A(x)$ for all $x \in G$. The converse is also true.

Corollary 4.4. Let $G$ be an abelian group. Let the function $f: G \rightarrow \mathbf{C}$ satisfy the functional equation

$$
f(x y)+f\left(y^{-1} x\right)=2 f(x)+2 f(y)
$$

for all $x, y \in G$. Then

$$
f(x)=B(x, x),
$$

where $B \in \operatorname{SBihom}(G \times G, \mathbf{C})$. The converse is also true. 
The following corollary follows from Corollary 4.2.

Corollary 4.5. Let $G$ be an abelian group and $\sigma: G \rightarrow G$ be an involutive endomorphism. Let the function $f: G \rightarrow \mathbf{C}$ satisfy the functional equation

$$
f(x y)+f\left(x y^{-1}\right)=2 f(x)+f(y)+f\left(y^{-1}\right)
$$

for all $x, y \in G$. Then

$$
f(x)=A(x)+B(x, x),
$$

where $B \in \operatorname{SBihom}(G \times G, \mathbf{C})$ and $A \in \operatorname{Hom}(G, \mathbf{C})$. The converse is also true.

\section{A generalization of (1.4)}

In the following theorem we present the solutions of a generalization of the functional equation (1.4) whose solutions were determined in Theorem 4.1.

Theorem 5.1. Let $G$ be a group and $\sigma_{1}, \sigma_{2}: G \rightarrow G$ be two endomorphisms satisfying $\sigma_{i}\left(\sigma_{i}(x)\right)=x$ for all $x \in G$ and for $i=1,2$. Assume $f: G \rightarrow \mathbf{C}$ is a central function. The functions $f, g, h, k: G \rightarrow \mathbf{C}$ satisfy the functional equation

$$
f(x y)+g\left(\sigma_{1}(y) x\right)=2 f(x)+h(y)+k\left(\sigma_{2}(y)\right)
$$

for all $x, y \in G$ if and only if

$$
\begin{aligned}
& f(x)=A(x)+B(x, x)+\beta, \\
& g(x)=A(x)+B(x, x)+\beta+\alpha, \\
& h(x)=\phi(x), \\
& k(x)=A\left(\sigma_{2}(x)\right)+A\left(\sigma_{1}\left(\sigma_{2}(x)\right)\right)+2 B\left(\sigma_{2}(x), \sigma_{2}(x)\right)+\alpha-\phi\left(\sigma_{2}(x)\right),
\end{aligned}
$$

where $\phi: G \rightarrow \mathbf{C}$ is a function, $B \in \operatorname{SBihom}(G \times G, \mathbf{C})$ satisfying $B\left(\sigma_{1}(x), y\right)=-B(x, y)$ for all $x, y \in G, A \in \operatorname{Hom}(G, \mathbf{C})$, and $\alpha, \beta$ are complex constants.

Proof. Letting $y=e$ in (5.1), we obtain

$$
g(x)=f(x)+\alpha
$$

where $\alpha:=h(e)+k(e)$ is a complex constant. Next, letting $x=e$ in (5.1), we have

$$
h(y)+k\left(\sigma_{2}(y)\right)=f(y)+g\left(\sigma_{1}(y)\right)-2 \beta,
$$


where $\beta:=f(e)$. Using (5.2) in the above expression, we get

$$
h(y)+k\left(\sigma_{2}(y)\right)=f(y)+f\left(\sigma_{1}(y)\right)+\alpha-2 \beta .
$$

Next, we substitute (5.2) and (5.3) into (5.1) to get

$$
f(x y)+f\left(\sigma_{1}(y) x\right)=2 f(x)+f(y)+f\left(\sigma_{1}(y)\right)-2 \beta
$$

for all $x, y \in G$. Defining a function $F: G \rightarrow \mathbf{C}$ by

$$
F(x)=f(x)-\beta
$$

for all $x \in G$. Then by (5.4), (5.5) reduces to

$$
F(x y)+F\left(\sigma_{1}(y) x\right)=2 F(x)+F(y)+F\left(\sigma_{1}(y)\right)
$$

for all $x, y \in G$. Hence from Corollary 4.2, we obtain the solution of the above functional equation as

$$
F(x)=A(x)+B(x, x)
$$

where $B \in \operatorname{SBihom}(G \times G, \mathbf{C})$ satisfying

$$
B\left(\sigma_{1}(x), y\right)=-B(x, y)
$$

for all $x, y \in G$ and $A \in \operatorname{Hom}(G, \mathbf{C})$. Using (5.7) in (5.5), we have

$$
f(x)=A(x)+B(x, x)+\beta
$$

and (5.9) in (5.2) yields

$$
g(x)=A(x)+B(x, x)+\beta+\alpha .
$$

From (5.3) and (5.9), we get $h(x)=\phi\left(\sigma_{2}(x)\right)$ and

$k(x)=A\left(\sigma_{2}(x)\right)+A\left(\sigma_{1}\left(\sigma_{2}(x)\right)\right)+2 B\left(\sigma_{2}(x), \sigma_{2}(x)\right)+\alpha-\phi\left(\sigma_{2}(x)\right)$.

It is easy to check that the solutions $(f, g, h, k)$ along with the condition (5.8) satisfy the functional equation (5.1). This finishes the proof of the theorem.

The following two corollaries follow from the above theorem. 
Corollary 5.2. Let $G$ be a group and $\sigma: G \rightarrow G$ be an endomorphism satisfying $\sigma(\sigma(x))=x$ for all $x \in G$. Assume $f: G \rightarrow \mathbf{C}$ is a central function. The functions $f, g: G \rightarrow \mathbf{C}$ satisfy the functional equation

$$
f(x y)+g(\sigma(y) x)=2 f(x)
$$

for all $x, y \in G$ if and only if

$$
f(x)=g(x)=A(x)+\beta,
$$

where $A \in \operatorname{Hom}(G, \mathbf{C})$ satisfying

$$
A(x)+A(\sigma(x))=0 \quad \forall x \in G .
$$

Corollary 5.3. Let $G$ be a group and $\sigma: G \rightarrow G$ be an endomorphism satisfying $\sigma(\sigma(x))=x$ for all $x \in G$. Assume $f: G \rightarrow \mathbf{C}$ is a central function. The functions $f, g, h: G \rightarrow \mathbf{C}$ satisfy the functional equation

$$
f(x y)+g(\sigma(y) x)=2 f(x)+h(y)
$$

for all $x, y \in G$ if and only if

$$
\begin{aligned}
& f(x)=A(x)+B(x, x)+\beta, \\
& g(x)=A(x)+B(x, x)+\beta+\alpha, \\
& h(x)=A(x)+A(\sigma(x))+2 B(x, x)+\alpha,
\end{aligned}
$$

where $A \in \operatorname{Hom}(G, \mathbf{C})$ and $B \in \operatorname{SBihom}(G \times G, \mathbf{C})$ satisfying

$$
B(\sigma(x), y)=-B(x, y)
$$

for all $x, y \in G$ and $\alpha, \beta$ are any complex constants.

\section{An application of (5.1)}

In the next theorem, as an application of Theorem 4.1, we determine the solution of a new functional equation (6.3) related to stochastic distance measures when $f$ is a central function. For other functional equations related to stochastic distance measures the interested reader should refer to $[3,16,17,19,20]$.

The following lemma is needed from [5] for the next theorem.

Lemma 6.1. Let $S$ be a monoid and $\sigma: S \times S \rightarrow S \times S$ the involutive endomorphism given $\sigma(p, q):=(q, p)$ for all $(p, q) \in S \times S$. Every symmetric 
bihomorphism $B:(S \times S) \times(S \times S) \rightarrow \mathbf{C}$ satisfying $B(\sigma(p, q),(r, s))=$ $-B((p, q),(r, s))$ for all $(p, q),(r, s) \in S \times S$ can be decomposed as

$$
B((p, q),(p, q))=\psi(p, p)-\psi(p, q)-\psi(q, p)+\psi(q, q)
$$

for all $p, q, r, s \in S$, where $\psi \in \operatorname{SBihom}(S \times S, \mathbf{C})$. In addition, if $S$ is a group, then

$$
B((p, q),(p, q))=\psi\left(p q^{-1}, p q^{-1}\right)
$$

for all $p, q, r, s \in S$.

Next, we present the main result of this section.

Theorem 6.2. Let $G$ be a group and $f: G \times G \rightarrow \mathbf{C}$ be a central function. The functions $f, g, h, k: G \times G \rightarrow \mathbf{C}$ satisfy the functional equation

$$
f(p r, q s)+g(s p, r q)=2 f(p, q)+h(r, s)+k(s, r)
$$

for all $p, q, r, s$ in $G$ if and only if $f, g, h, k$ are functions of the form

$$
\begin{gathered}
f(p, q)=\theta_{1}(p)+\theta_{2}(q)+\psi\left(p q^{-1}, p q^{-1}\right)+\beta, \\
g(p, q)=\theta_{1}(p)+\theta_{2}(q)+\psi\left(p q^{-1}, p q^{-1}\right)+\beta+\alpha, \\
h(p, q)=\phi(p, q), \\
k(p, q)=\theta_{1}(p q)+\theta_{2}(p q)+2 \psi\left(p q^{-1}, p q^{-1}\right)-\phi(q, p)+\alpha,
\end{gathered}
$$

where $\theta_{1}, \theta_{2} \in \operatorname{Hom}(G, \mathbf{C}), \psi \in \operatorname{SBihom}(G \times G, \mathbf{C}), \phi: G \times G \rightarrow \mathbf{C}$ is any function, and $\alpha, \beta \in \mathbf{C}$ are constants.

Proof. It easy but tedious to check that the functions $(f, g, h, k)$ given by (6.4), (6.5), (6.6), (6.7) satisfy the functional equation (6.3). Next, we show that the functions $(f, g, h, k)$ given by $(6.4),(6.5),(6.6),(6.7)$ constitute the only solution of (6.3).

Let $(f, g, h, k)$ be a solution of the functional equation (6.3). Define a function $\sigma: G \times G \rightarrow G \times G$ such that $\sigma(p, q)=(q, p)$ for all $p, q \in G$. Then $\sigma$ is an endomorphism and it is also involutive, that is

$$
\sigma(\sigma(p, q))=\sigma(q, p)=(p, q)
$$

for all $p, q \in G$. Let $x=(p, q)$ and $y=(r, s)$ in (6.3). Then it reduces to (1.4) for all $x, y \in G \times G$. Since $f$ is central, from Theorem 5.1 with $\sigma_{1}=\sigma_{2}=\sigma$, we obtain 


$$
\begin{aligned}
& f(x)=A(x)+B(x, x)+\beta, \\
& g(x)=A(x)+B(x, x)+\beta+\alpha, \\
& h(x)=\phi(x), \\
& k(x)=A(\sigma(x))+A(x)+2 B(\sigma(x), \sigma(x))-\phi(\sigma(x))+\alpha,
\end{aligned}
$$

where $B \in S$ Bihom $((G \times G) \times(G \times G), \mathbf{C})$ satisfying $B(\sigma(x), y)=-B(x, y)$ for all $x, y \in G \times G, A \in \operatorname{Hom}(G \times G, \mathbf{C}), \phi: G \times G \rightarrow \mathbf{C}$ is any function, and $\alpha, \beta$ are complex constants. Since $x \in G \times G$, we can write $x=(p, q)$ for $p, q \in G$. Hence we have

$$
\begin{aligned}
& f(p, q)=A(p, q)+B((p, q),(p, q))+\beta \\
& g(p, q)=f(p, q)+\alpha \\
& h(p, q)=\phi(p, q) \\
& k(p, q)=A(q, p)+A(p, q)+2 B((q, p),(q, p))-\phi(q, p)+\alpha
\end{aligned}
$$

for all $p, q \in G$. Using Lemma 5.2 from [4], the functions $A$ can be decomposed as $A(p, q)=\theta_{1}(p)+\theta_{2}(q)$, where $\theta_{1}, \theta_{2} \in \operatorname{Hom}(G, \mathbf{C})$. Using Lemma 6.1, the bihommorphism $B$ can be further simplified as

$$
B((p, q),(p, q))=\psi\left(p q^{-1}, p q^{-1}\right) .
$$

Inserting the form of $A(p, q)$ and $B((p, q),(p, q))$ for $(f, g, h, k)$ we have the asserted solutions (6.4)-(6.7). The proof of the theorem is now complete.

\section{Acknowledgment}

The author is very thankful to the referee for valuable suggestions that improved the readability. This work was partially supported by a research grant from the Office of the Executive Vice President for Research and Innovation, University of Louisville.

\section{References}

[1] J. Aczél, The general solution of two functional equations by reduction to functions additive in two variables and with aid of Hamel bases, Glasnik Mat.-Fiz. Astronom. Drustvo Mat. Fiz. Hrvatske, 20, pp. 65$73,(1965)$.

[2] J. Aczél and E. Vincze, Über eine gemeinsame Verallgemeinerung zweier Funktionalgleichungen von Jensen, Publ. Math. Debrecen, 10, pp. 326-344, (1963). 
[3] J. K. Chung, Pl. Kannappan, C. T. Ng, and P. K. Sahoo, Measures of distance between probability distributions, J. Math. Anal. Appl., 139, pp. 280-292, (1989).

[4] H. H. Elfen, T. Riedel and P.K. Sahoo, A variant of quadratic functional equation on groups. Submitted, (2016).

[5] H. H. Elfen, T. Riedel and P.K. Sahoo, A variant of a generalized quadratic functional equation on groups, To appear in Results in Math., (2017).

[6] V. A. Faiziev and P. K. Sahoo, Solution of Whitehead equation on groups, Math. Bohemica, (2) 138 (2013), 171-180.

[7] G. L. Forti, Stability of quadratic and Drygas functional equations, with an application for solving alternative quadratic equation, T.M. Rassias (ed.), Handbook of Functional Equations, Springer Optimization and Its Applications 96, DOI 10.1007/978-1-4939-1286-5-8.

[8] J. L. W. V. Jensen, On the solution of fundamental equations by elementary means (Danish), Tidsskr. Math., 4, pp. 149-155, (1878).

[9] J. L. W. V. Jensen, On the solution of functional equations with the minimal numbers of suppositions (Danish), Mat. Tidsskr. B, pp. 25-28, (1897).

[10] P. Jordan and J. von Neumann, On the inner products in linear metric spaces, Ann. Math., 36, pp. 719-723, (1935).

[11] Pl. Kannappan, Functional Equations and Inequalities with Applications, Singapore, pp. 249, (2009).

[12] Pl. Kannappan, On inner product spaces, I, Math. Jpn., (2) 45, pp. 289-296, (1997).

[13] Pl. Kannappan, On quadratic functional equation, Int. J. Math. Statist. Sci., 9, pp. 35-60, (2000).

[14] S. Kurepa, On the quadratic functional, Publ. Inst. Math. Acad. Serbe Sci., 13, pp. 58-78, (1959).

[15] C. T. Ng and H. Y. Zhao, Kernel of the second order Cauchy difference on groups, Aequat. Math., 86, pp. 155-170, (2013). 
[16] T. Riedel and P. K. Sahoo, On two functional equations connected with the characterizations of the distance measures, Aequat. Math., 54, pp. 242-263, (1997).

[17] T. Riedel and P. K. Sahoo, On a generalization of a functional equation associated with the distance between the probability distributions, Publ. Math. Debrecen, 46, pp. 125-135, (1995).

[18] P. K. Sahoo, The Drygas functional equation with involution on groups, Submitted, (2016).

[19] P. K. Sahoo, On a functional equation associated with stochastic distance measures, Bull. Korean Math. Soc., 36, pp. 287-303, (1999).

[20] P. K. Sahoo and Pl. Kannappan, Introduction to Functional Equations, Chapman and Hall/CRC, pp. 269-290, (2011).

[21] P. Sinopoulos, Functional equations on semigroups, Aequat. Math., 59, pp. 255-261, (2000).

[22] H. Stetkaer, Functional Equations on Groups, World Scientific Publishing, Singapore (2013).

[23] H. Stetkaer, A variant of d'Alembert's functional equation, Aequat. Math., 89, pp. 657-662, (2015).

[24] D. Yang, The quadratic functional equation on groups, Publ. Math. Debrecen, (3) 66, pp. 327-348, (2004).

[25] J. H. C. Whitehead, A certain exact sequence, Ann. Math., (2), 52, pp. 51-110, (1950).

\author{
Prasanna K. Sahoo \\ Department of Mathematics, \\ University of Louisville, \\ Louisville, \\ Kentucky 40292 \\ U.S.A. \\ e-mail : sahoo@louisville.edu
}

